Original Research Article

\title{
Antibiotic sensitivity pattern to urinary tract infections in a tertiary care hospital in South India
}

\author{
Shanmugapriya S. ${ }^{1 *}$, Saravanan T. ${ }^{2}$, Janani K. ${ }^{3}$
}

\begin{abstract}
${ }^{1}$ Department of Pharmacology, ${ }^{2}$ Department of Medicine, ${ }^{3}$ Final year MBBS student, PSG Institute of Medical Sciences and Research, Coimbatore 641004, Tamilnadu, India

Received: 30 March 2017 Accepted: 27 April 2017

\section{*Correspondence to: \\ Dr. Shanmugapriya S., Email: somasundaram999@ rediffmail.com}

Copyright: (C) the author(s), publisher and licensee Medip Academy. This is an openaccess article distributed under the terms of the Creative Commons Attribution NonCommercial License, which permits unrestricted noncommercial use, distribution, and reproduction in any medium, provided the original work is properly cited.

\begin{abstract}
Background: Urinary tract infection (UTI) is one of the most common bacterial illnesses and hence one of the most important indications for antibiotic treatment. Current knowledge of the common organisms implicated in causing UTI in the local community and surveillance to monitor the changes in susceptibility of uropathogens are imperative to ensure appropriate therapy. The study objectives were to assess the proportion of UTI caused by each of the common urinary pathogens, to study the antibiotic drug sensitivity patterns by analyzing the culture and sensitivity reports and to identify the drugs which would be potentially favourable candidates for empirical therapy in the study locale.

Methods: This hospital based cross sectional study was done by collecting the data from case records of patients who attended OPD or were admitted with diagnosis of UTI during the study period.

Results: Escherichia coli was the single most important cause of UTI, accounting for $70 \%$ of the infection among the studied subjects, amongst which $55 \%$ was extended spectrum beta-lactamase producing organisms. A pattern of increased susceptibility to higher antibiotics like ertapenem, tigecycline and decreased sensitivity to fluroquinolones was evident. In addition, nitrofurantoin was found to be one of the antibiotics to which most of the organisms were susceptible (85\%) favouring its use in empirical therapy of UTI.

Conclusions: The pattern of accentuated susceptibility to higher antibiotics in the face of declining trend in the sensitivity to commonly used antibiotics necessitates promoting awareness on the need for rational prescribing of antibiotics among clinicians.
\end{abstract}

Keywords: Antibiotics, Culture and sensitivity, Escherichia coli, Urinary tract infection

\section{INTRODUCTION}

Urinary tract infections (UTI) remain one of the commonest infections diagnosed in out-patients and hospitalized patients. An in-depth knowledge on antimicrobial susceptibility pattern is essential for appropriate therapy, particularly with increasing resistance resulting from repeated use of the same antibiotic. ${ }^{1}$ It is also essential to be aware of the changing patterns of antibiotic resistance in a locale. ${ }^{2}$ Published data may not always represent the scenario prevailing in a particular location as the studies done elsewhere could vary in the pattern of microorganisms involved in causation, their sensitivity and resistance to different antibiotics which in turn depends on the antibiotic prescribing pattern in that region. This indicates that there is a need for studying the existing antibiotic sensitivity patterns in the local population. ${ }^{3}$ Resistance significantly varies by region and the highest antibiotic resistance has been observed in developing countries. Similarly a significant rise in resistance over time is seen in studies reporting on community acquired $E$. coli $\mathrm{UTI}^{3}$

Hence this study was designed to evaluate the drug sensitivity patterns to some of the commonly used antimicrobials in UTI like the beta lactams, fluroquionolones and aminoglycosides. In addition, the 
antibacterial drugs commonly used in UTI like nitrofurantoin, cotrimoxazole were also studied for the sensitivity patterns. Thus, the study was done to provide an insight into the drug sensitivity patterns of common urinary pathogens to antibiotic therapy by analyzing the culture and sensitivity reports.

Our study was designed to identify the proportion of UTI caused by each of the urinary pathogens prevalent in the study locale and the likely drug candidates with the potential of being used as empirical therapy in community acquired pathogens by evaluating their sensitivity pattern, thereby enabling identification of the drugs which can effectively be used in the management of UTI.

\section{METHODS}

This was a hospital based study done at a tertiary care teaching hospital after the approval from Institutional Human Ethics Committee. The study was a cross sectional one, done using case records. The data from case records of the patients who attended OPD or admitted with diagnosis of UTI during the study period were included for the study based on inclusion and exclusion criteria.

The data from the case records of adult patients above the age of 20 years, of both sexes, diagnosed with UTI were included for the study. Patients with asymptomatic bacteriuria, those with complicated UTI, upper UTI or known malformations, paediatric and pregnant patients, immunocompromised patients like patients with HIV, on corticosteroids or immunosuppressant therapy, organ transplant recipient were excluded.

The culture and in vitro sensitivity testing report data were collected in addition to demographic data, co morbid conditions, symptoms and duration of symptoms and drug therapies. In addition, data on history of hospitalizations, catheterizations and frequency of recurrences were also obtained from the case sheets. Also, the details recorded on the review date regarding cure or continuing symptoms, repeat culture reports, if any, was made note of from the case record.

\section{RESULTS}

A total of 20 patients whose case records indicated that they had been diagnosed with UTI were included for the study. All these patients were urine culture positive. In our study $30 \%$ of patients were in the age group $20-40$ years and a large majority of about $45 \%$ were in the age group of 41-60 years and $25 \%$ of the patients were aged 61 -80 years (Figure 1). Male female ratio in our study population was $65: 35$.

Catheterization was done in $20 \%$ of the study subjects. The duration of catheterization was variable with one patient having a history of prolonged catheterization of
12 days. Concomitant renal disease was found in a high proportion of the UTI patients. Amongst the studied population, $10 \%$ had renal failure, $10 \%$ nephritis and $5 \%$ pyelonephritis with renal calculi. One patient manifested with lupus nephritis as she was a known case of systemic lupus erythematosis. One patient was a case of neurogenic bladder. About $10 \%$ patients had presented with concomitant prostatitis and a similar $10 \%$ with UTI had history of recurrent episodes (Figure 2).

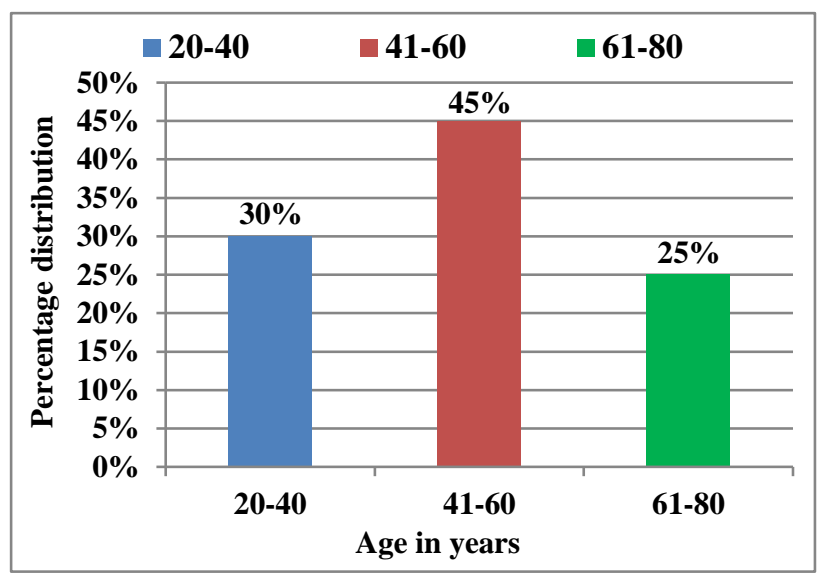

Figure 1: Frequency distribution of age in the study population.

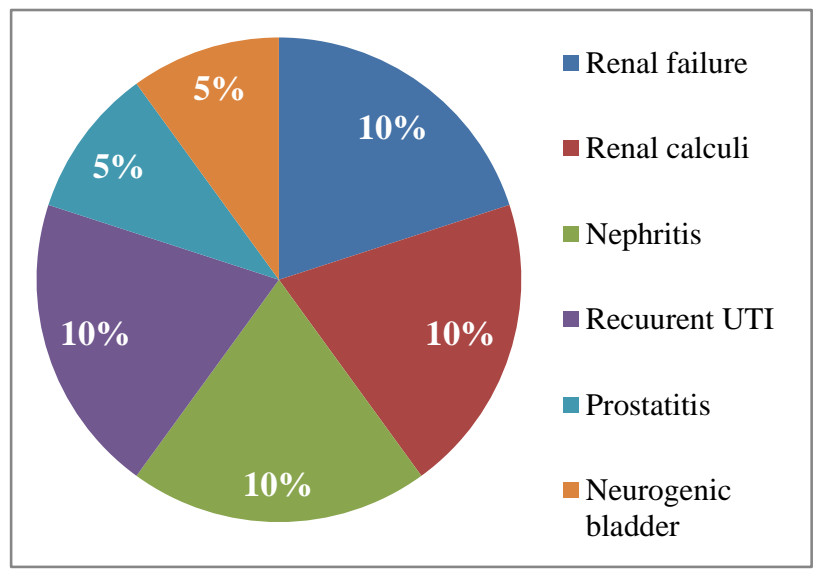

Figure 2: Frequency distribution of concomitant renal disease.

Data also demonstrated that concomitant systemic diseases were found in a significant proportion of the patients whose records were evaluated. A total of $40 \%$ had diabetes mellitus and $30 \%$ patients were hypertensives. Interestingly, amongst the $30 \%$ with hypertension, $83 \%$ were both diabetic and hypertensives. Heart disease was seen in $10 \%$, one with diastolic dysfunction and the others with coronary artery disease. Amongst those whose data were studied, it was found that there were about five patients with other chronic systemic illnesses like chronic calcific pancreatitis, pituitary ademona, hypothyroidism, SLE, liver disease with cholelithiasis (Figure 3). 


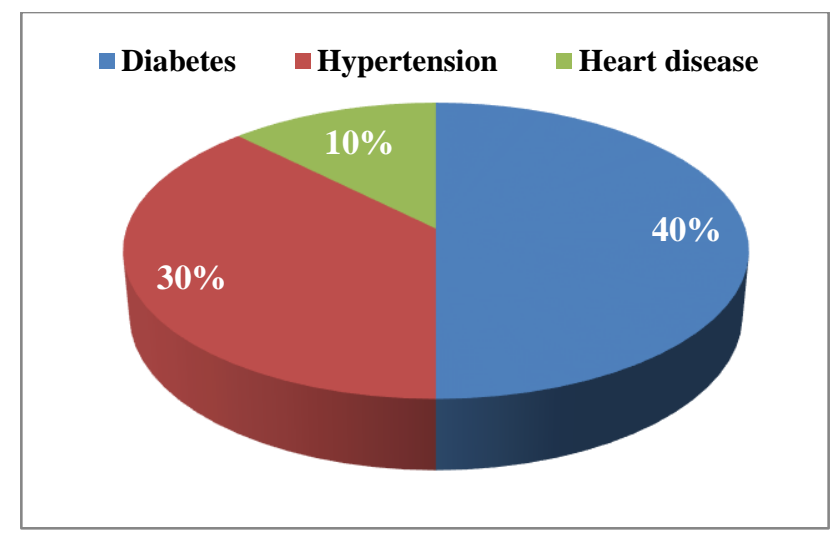

Figure 3: Frequency distribution of concomitant systemic diseases.

Escherichia coli was the single most important microorganism found to implicated in causation of UTI, accounting for $70 \%$ of the infection among the studied subjects (Table 1).

Table 1: Percentage distribution of organisms isolated in culture.

\begin{tabular}{|ll|}
\hline Organisms grown in culture & Percentage \\
\hline Escherichia coli (ESBL) & $55 \%$ \\
\hline Escherichia coli & $15 \%$ \\
\hline Pseudomonas aeroginosa & $10 \%$ \\
\hline Enterococcus fecium & $10 \%$ \\
\hline Enterococcus fecalis & $5 \%$ \\
\hline Klebsiella pneumoniae & $5 \%$ \\
\hline Infection with dual organisms & $10 \%$ \\
\hline
\end{tabular}

Organisms grown in urine culture in the study population revealed a high proportion of $E$. coli being Extended spectrum beta-lactamase (ESBL) producing organisms. Next in frequency was Pseudomonas aeroginosa and Enterococcus fecium, responsible for $10 \%$ each whilst Enterococcus fecalis and Klebsiella contributing 5\% each. Co-infection with two of these organisms (dual infection) was seen in $10 \%$ (Figure 4).

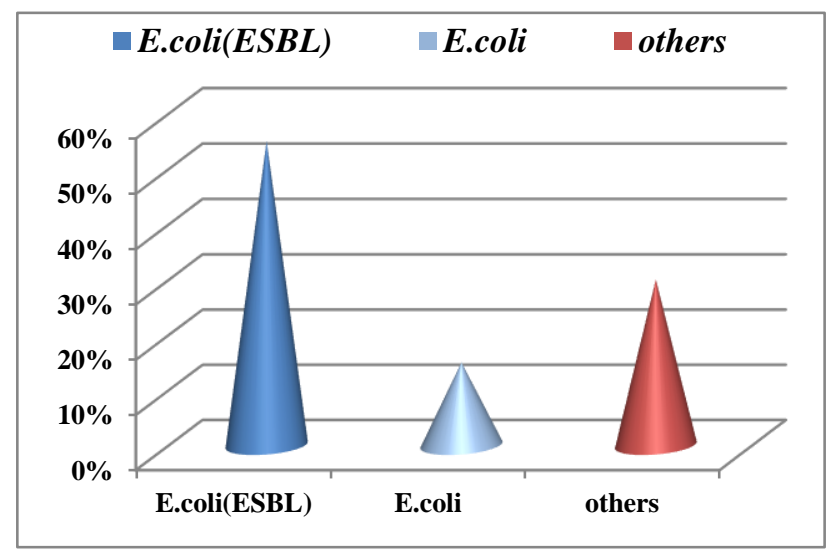

Figure 4: Frequency distribution of the urinary pathogens isolated in culture.
The sensitivity of the cultured organisms was also evaluated based on their susceptibility to different groups of antibiotics.

\section{Aminoglycosides}

The sensitivity to amikacin was found to be highest $(60 \%)$ followed by netilmycin $(40 \%)$ and the sensitivity for tobramycin and gentamycin were equal, each with $30 \%$ (Figure 5).

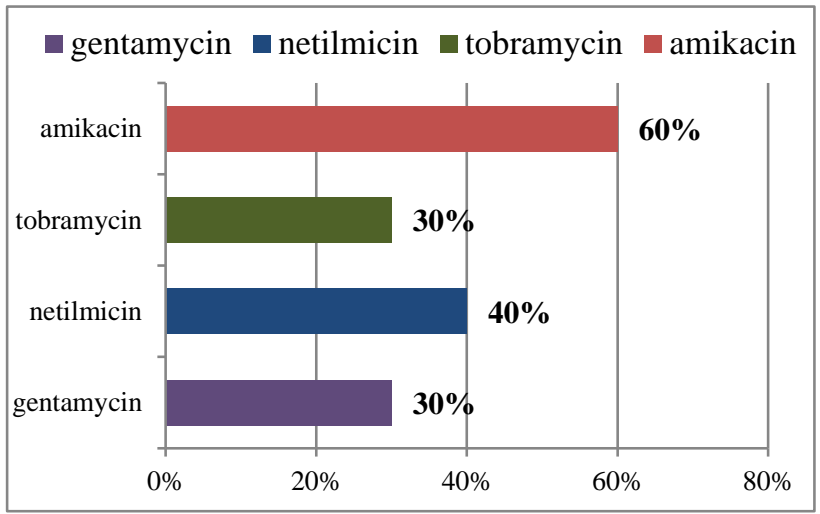

Figure 5: Frequency distribution of sensitivity to aminoglycosides.

\section{Fluoroquinolones}

The overall sensitivity to quinolones was found to be lower than that of aminoglycosides and among the quinolones, the greatest sensitivity percentage was recorded for ofloxacin (Figure 6).

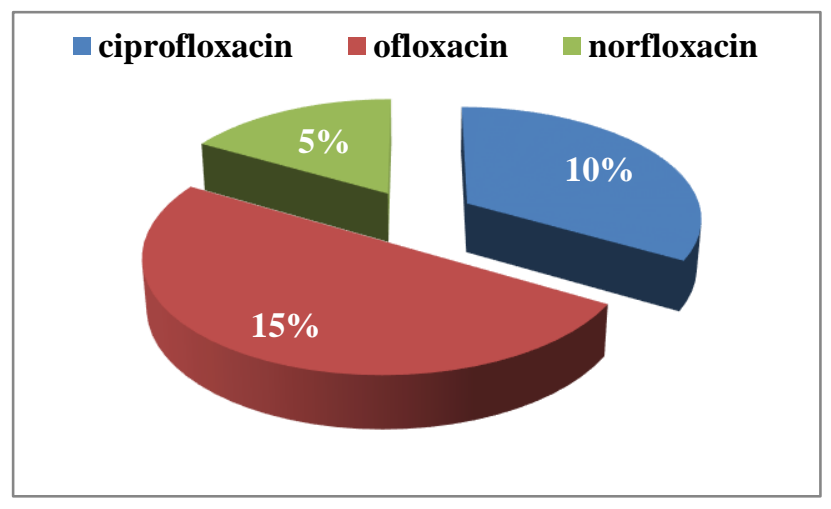

Figure 6: Frequency distribution of sensitivity to fluoroquinolones.

\section{Beta lactams}

Analyzing beta-lactams demonstrating high sensitivity, it was found that remarkably higher sensitivity was seen for beta lactams when combined with beta-lactamase inhibitors. It is quite obvious that the greater susceptibility can be accounted by the high proportion of ESBL organisms cultured. $60 \%$ of all culture reports studied revealed sensitivity of the cultured bacteria to 
cefaperazone in combination with sulbactam and the next highest being piperacillin tazobactum combination with $45 \%$ and a slightly lower percentage of $40 \%$ was recorded for amoxicillin clavulanate (Figure 7).

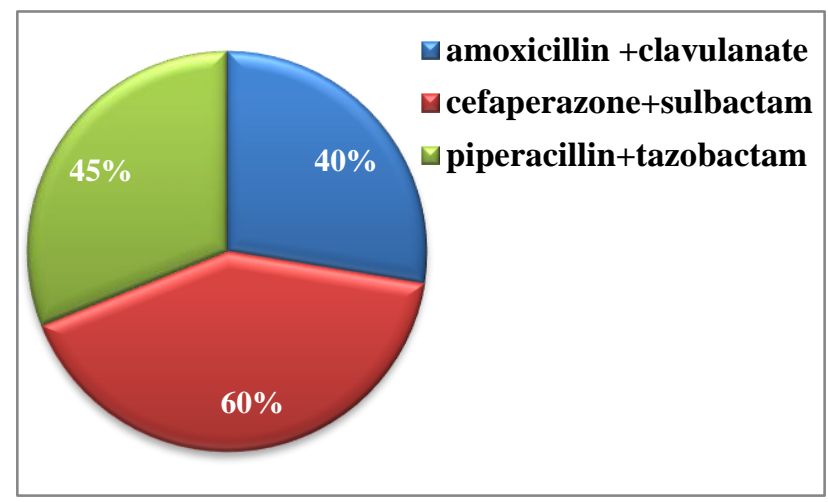

Figure 7: Frequency distribution of sensitivity to commonly used beta lactams in combination with beta-lactamase inhibitors.

\section{Amino-penicillins}

The aminopenicillins were one of the drug groups studied for bacterial sensitivity apart from ureidopencillins in the penicillin group of beta-lactam antibiotics and among the two amino-pencillins used commonly, organisms showed a marginally higher sensitivity to ampicillin (20\%) as compared to amoxicillin (10\%).

\section{Cephalosporins}

In the cephalosporin group, cefaperazone in combination with sulbactum was the antibiotic to which most organisms $(60 \%)$ were sensitive. Apart from it, the other cephalosporins found to be useful in $10-20 \%$ of infections based on culture and sensitivity pattern are cefepime, ceftriaxone, cephalothin and cefuroxime respectively with $30 \%, 20 \%, 15 \%$ and $10 \%$. It was evident that sensitivity for the first and second generation was lower in comparison to third and fourth generations of cephalosporins (Figure 8).

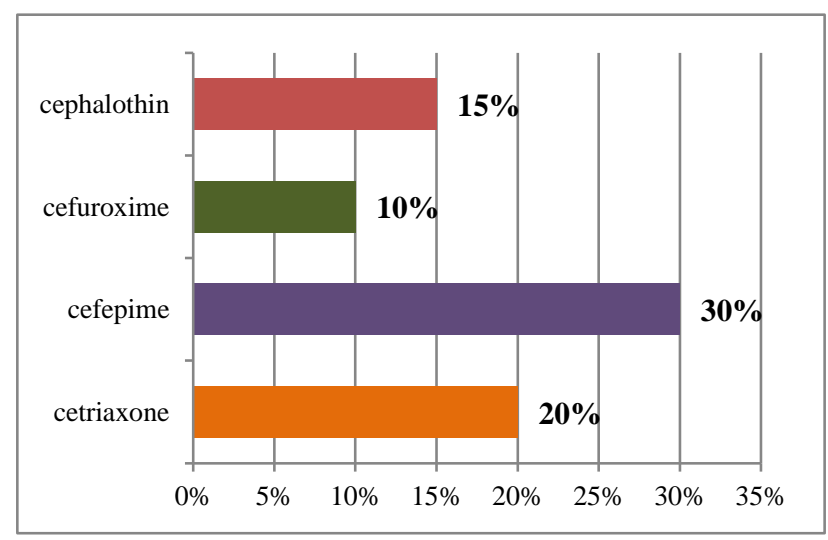

Figure 8: Frequency distribution of sensitivity to cephalosporins.

\section{Carbapenems}

Among carbapenems, greatest sensitivity was recorded for ertapenem (70\%) (Figure 9).

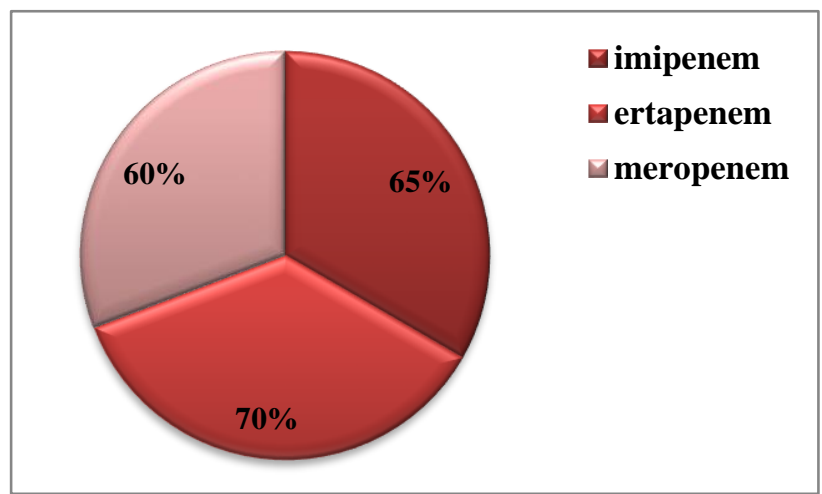

Figure 9: Frequency distribution of sensitivity to carbapenems.

\section{Tetracycline and glycylcycline}

Tigecycline was found to be the antibiotic to which $60 \%$ of the organisms studied were susceptible to and it matched that of cefaperazone combined with sulbactum in achieving the same percentage of sensitivity (Figure $10)$.

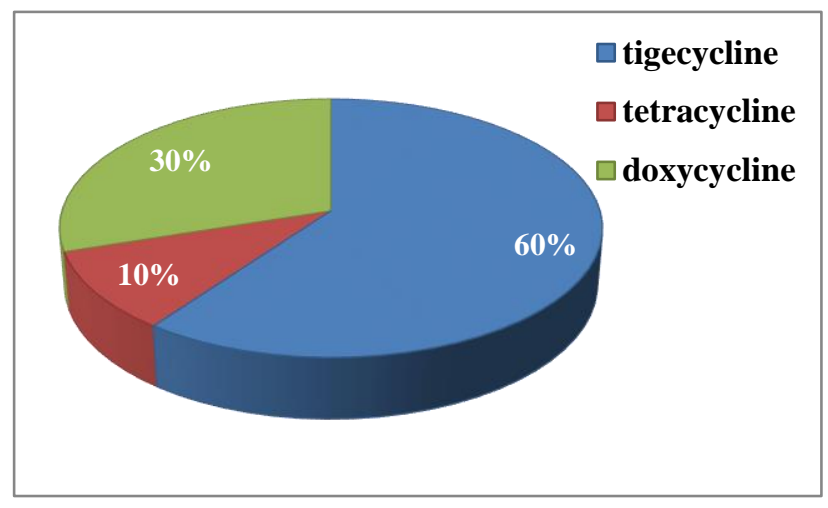

Figure 10: Frequency distribution of sensitivity to tetracyclines and glycylcycline.

\section{Miscellaneous antibacterials}

Interestingly, $85 \%$ of the cultures showed sensitivity to nitrofurantoin. Also, colistin was another antibiotic found to have similar percentage of sensitivity comparable with tigecycline and cefaperazone sulbactam combination. Sensitivity to cotrimoxazole was $40 \%$ and that for vancomycin and linezolid, with $20 \%$ each (Figure 11).

The pattern of antibiotic sensitivity of the UTI causing organisms was analyzed. Comparing the various drug groups with differential sensitivity percentages, the highest in-vitro sensitivity was to the beta lactam group followed by nitrofuantoin and colistin of the 
miscellaneous antibacterial group and the least sensitivity was demonstrated by the fluoroquinolones (Figure 12).

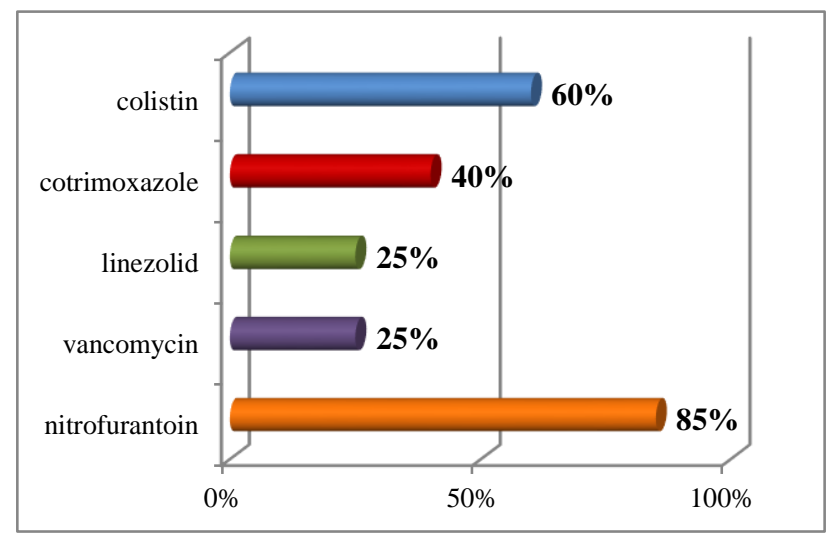

Figure 11: Frequency distribution of sensitivity to miscellaneous antibiotics.

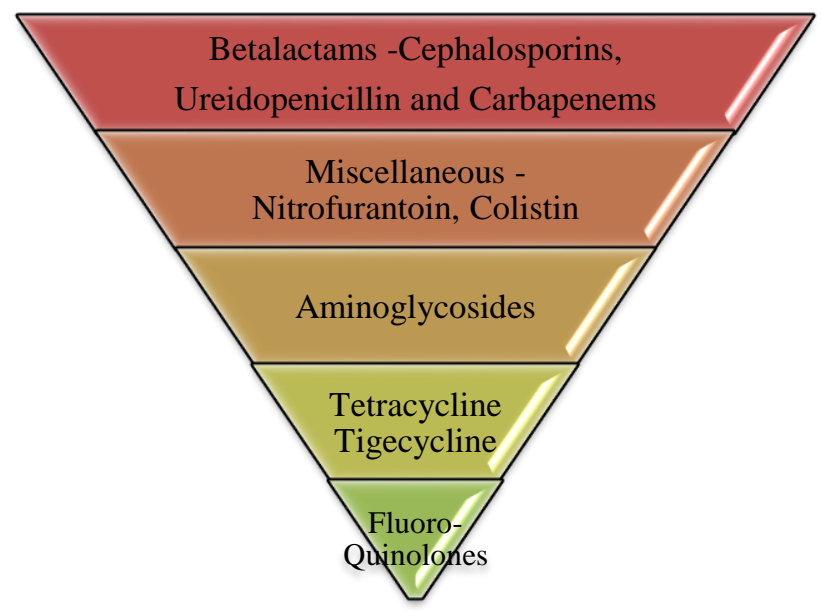

Figure 12: The pattern of drug susceptibility of urinary pathogens to different antibiotics.

\section{DISCUSSION}

Urinary tract infections (UTI) is the most common infection experienced by human after respiratory and gastrointestinal infections, accounting for 8.6 million visits ( $84 \%$ by women) in 2007 in the United States., ${ }^{4,5}$ UTI is said to occur more commonly in females than in males; in males it is uncommon, but often associated with structural abnormality. ${ }^{6}$ But in our sample, we found a greater percentage of males than females.

The highest co-morbidity noted in our study population was diabetes and hypertension. Diabetes plays a definitive role in increasing the occurrence of UTI because of the changes in immunological function and thereby increasing the susceptibility to UTI. Studies have demonstrated that the epidemiology of urinary tract infections (UTIs) among men and women with diabetes is similar to the epidemiology of those without diabetes with women having greater risk than men. ${ }^{7}$ However, the bacteriology and antibiotic susceptibility patterns also do not, in general, differ from those without diabetes. ${ }^{7,8}$ Our study results are well in line with these findings. In addition, a study has revealed that the virulence factors of $E$. coli affecting the diabetic individuals also remains the same as in the general population. ${ }^{8}$

Worldwide, the most frequent etiology is Escherichia coli and this is also reflected in our study results. ${ }^{9}$ However the sensitivity and resistance patterns of $E$. coli strains causing UTI varies considerably between regions and countries. ${ }^{10}$ In this study, we found that most E. coli isolated in culture were susceptible to cefaperazonesulbactum, ertapenem, tigecycline, nitrofurantoin, colistin and amikacin. Among these nitrofurantoin and colistin are the drugs administered orally and the rest have to be administered by parenteral route. Interestingly, we found a decreasing sensitivity to fluoroquinolones which have been regarded as one of the first line drugs in UTI and therefore rampantly used which consecutively would have lead to a high degree of fluoroquinolone resistance in our population.

The study highlights the increasing trend of resistant Gram negative organism in culture necessitating the need for higher antibiotics. A study has highlighted that among tetracyclines, tigecycline has best in vitro susceptibility against ESBL producing Gram negative rods. ${ }^{11}$ Likewise, a high cure rate, both clinical and microbiological has been recorded for ertapenem also, particularly for ESBL infections. ${ }^{12}$ The indiscriminate use of such higher antibiotics like ertapenem and tigecycline will foster development of drug resistance, further complicating the management of urinary tract infection.

The vicious cycle wherein widespread use of newer antibiotics leading on to the organisms developing resistance to them and that in sequence, further necessitating the development of newer antibiotics ultimately results in difficult to treat infections. Such a situation can be overcome by implementing rational prescribing practices like the use of lower antibiotics with limited spectrum to which the organism is susceptible rather than resorting to prescribe higher antibiotics with a wide spectrum. ${ }^{13}$

This study has clearly delineated the emphatic role of nitrofurantoin in urinary tract infections. Our findings are in concordance with currently emerging trend in the use of nitrofurantoin as a key oral antibiotic option in the treatment of acute uncomplicated cystitis due to multidrug resistant Gram negative bacilli causing UTI. ${ }^{14}$ Studies also have acknowledged that the efficacy of nitrofurantoin for the empirical treatment is supportable from a public health perspective in an attempt to decrease uropathogen resistance. ${ }^{15}$ Thus the current scenario based on drug sensitivity justifies the use of nitrofurantoin in the empiric management of UTI, not only due to the high susceptibility of E. coli to nitrofurantoin and the ease of oral administration but also owing to the fact that it lacks 
cross-resistance with commonly prescribed antibiotics for UTI. However, nitrofurantoin is contraindicated in renal disease since most of it is really excreted.

\section{Limitation}

The limitation of the study is the small sample size. A larger study with a larger sample is needed to confirm the drug sensitivity pattern of UTI causing organisms in the study locale established through our study results.

\section{CONCLUSION}

The study highlights that $E$. coli contributes to the majority of the urinary tract infections diagnosed in our tertiary care hospital. In addition, the current drug sensitivity pattern signifies the need for higher antibiotics and this mandate implementing strategies of rational prescribing to avert forging resistance to these agents also. Nitrofurantoin, with good activity against a high percentage of the cultured organisms causing UTI, rationalizes its use for empirical treatment of UTI.

Funding: No funding sources Conflict of interest: None declared

Ethical approval: The study was approved by the Institutional Human Ethics Committee (Approval number: 16/158)

\section{REFERENCES}

1. Akram M, Shahid M, Khan AU. Etiology and antibiotic resistance patterns of community acquired urinary tract infections in JNMC Hospital, Aligarh, India. Ann Clinical Antimicrob, 2007 Mar 23;6:4.

2. Fasugba O, Gardner A, Mitchell BG, Mnatzaganian G. Ciprofloxocin resistance in community and hospital acquired Escherichia coli urinary tract infections: a systematic review and meta analysis of observational studies. BMC Infec Dis, 2015 Nov 25;15(1):545.

3. Cullen IM, Manecksha RP, McCullagh E, Ahmad S, O'Kelly F, Flynn RJ, et al. The changing pattern of antimicrobial resistance within 42,033 Escherichia coli isolates from nosocomial, community and urology patient-specific urinary tract infections, Dublin, 1999-2009. BJU Int. 2012 Apr;109(8):1198206.

4. Najar MS, Saldanha CL, Banday KA. Approach to urinary tract infections. Indian J Nephrol. 2009 Oct;19(4):129-39.
5. Hooton TM. Clinical practice. Uncomplicated urinary tract infection. N Engl J Med. 2012;366:1028-37.

6. Ferdosi-Sh E, Javanian M, Moradian KM. Resistance patterns of Escherichia coli causing urinary tract infection Caspian J Intern Med. 2015;6(3):148-51.

7. De Lastours V, Foxman B. Urinary tract infection in diabetes: epidemiologic considerations. Curr Infect Dis Rep. 2014 Jan;16(1):389.

8. Harwalkar A, Gupta S, Rao A, Srinivasa H. Prevalence of virulence factors and phylogenetic characterization of uropathogenic Escherichia coli causing urinary tract infection in patients with and without diabetes mellitus. Trans R Soc Trop Med Hyg. 2015 Dec;109(12):769-74.

9. Flores-Mireles AL, Walker JN, Caparon M, Hultgren SJ. Urinary tract infections: epidemiology, mechanisms of infection and treatment options. Nat Rev Microbiol. 2015 May;13(5):269-84.

10. Ferdosi-Shahandashti E, Javanian M, MoradianKouchaksaraei M, Yeganeh B, Bijani A, Motevaseli E, et al. Resistance patterns of Escherichia coli causing urinary tract infection. Caspian J Intern Med. 2015;6(3):148-51.

11. Gill MM, Usman J, Hassan A, Kaleem F, Shaheen N. In vitro susceptibility pattern of extended spectrum $\beta$ lactamase producing gram negative bacilli against tetracyclines. J Ayub Med Coll Abbottabad. 2015 Oct-Dec;27(4):788-90.

12. Veve MP, Wagner JL, Kenney RM, Grunwald JL, Davis SL. Comparison of fosfomycin to ertapenem for outpatient or step-down therapy of extendedspectrum $\beta$-lactamase urinary tract infections. Int $\mathbf{J}$ Antimicrob Agents. 2016 Jul;48(1):56-60.

13. Beveridge LA, Davey PG, Phillips G, McMurdo ME. Optimal management of urinary tract infections in older people. Clin Interv Aging. 2011;6:173-80.

14. Cunha BA, Cunha CB, Lam B, Giuga J, Chin J, Zafonte VF, Gerson S. Nitrofurantoin safety and effectiveness in treating acute uncomplicated cystitis (AUC) in hospitalized adults with renal insufficiency: antibiotic stewardship implications. Eur J Clin Microbiol Infect Dis. 2017 Feb 2.

15. Hooton TM. The current management strategies for community-acquired urinary tract infection. Infect Dis Clin North Am. 2003 Jun;17(2):303-32.

Cite this article as: Shanmugapriya S, Saravanan T, Janani K. Antibiotic sensitivity pattern to urinary tract infections in a tertiary care hospital in South India. Int J Basic Clin Pharmacol 2017;6:1445-50. 